\title{
Advanced Free Form Manufacturing by Computer Aided Systems - Cax
}

\author{
Adriano Fagali De Souza and Sabrina Bodziak \\ PROMOLDE Research Group, \\ Tupy Superior Institute (IST/SOCIESC), Santa Catarina, S.C., \\ Brazil
}

\section{Introduction}

As part of an integrated system technology CIM (Computer Integrated Manufacturing), longed for since the 50's, the computational systems known the CAx (Computer Aided specific manufacturing phase) developed to help phases of the manufacturing of a product and its components. In order to complete integration of the chain of manufactures by computer, CAx can be integrated with other computational systems of management and planning of trials and output, such as MRP (Material Resource Planning), ERP (Enterprise Resource Planning), EDM (Electronic Document Management)/PDM (Product Dates Management).

In this context, this chapter discusses the computational CAx systems to aid the activities related to the manufacturing, in special, the manufacturing of free form geometries. Such geometries are frequently found in the industry automobile, industry of moulds and dies, aeronautic and need CAx systems for its making. Machines controlled by Computer Numerical Command (CNC) for making parts and prototypes as well as for aiding geometrical inspection, also take part on the family of CAx systems and are discussed in this chapter.

This chapter includes: i) a brief definition about free form geometries; ii) main concerns about the manufacturing process of free form geometries; iii) CAx for free form manufacturing; iv) advanced features of CAx for free form design and manufacturing.

\section{A definition about free form geometries}

First, a definition of free form geometry (also known as sculptured surface) has to be set: it is a non-linear and curved shape, with neither constant nor pattern curvature radius along the geometry

Initially these geometric forms were employed exclusively in situations where they were to be used such as the aeronautical and naval industry. Currently complex geometric forms are becoming popular and can be found in various components in the automobile industry; in the consumption industry; toys, packaging, electronic products, esthetical products and ergonomics. This definition is important once that any manufacturing planning depends upon its shape. For instance, rotational parts have to be turned and prismatic parts have to be milled. Free form geometry has its own particularity to be manufactured. 


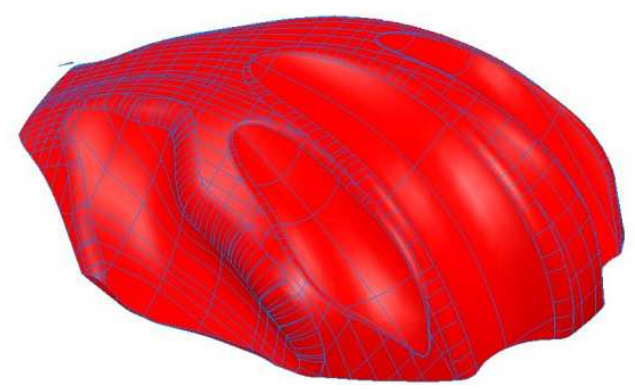

(a) Product free form shape

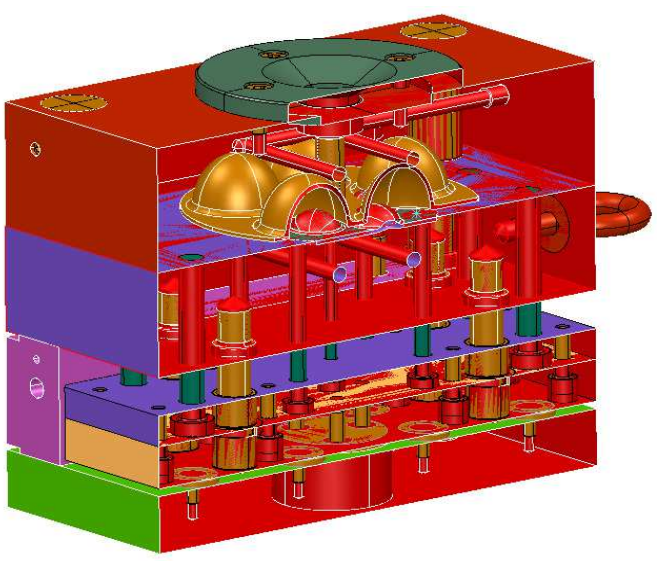

(b) Mould for plastic product

Fig. 1. Free form geometries

To represent computationally these sorts of geometry higher order polynomials are required. These equations were developed by Pierre Bézier, an French engineer who based on a Hermite proposal implemented the software Unisurf, in 1972. Such polynomials are known as Spline. The equation 1 is the Spline defined by Bézier. The curve is guided by a polygon (SOUZA et al, 2010). Using a mathematical software the Figure 2 was plotted.

$$
p(u)=p_{0}\left(1-3 u+3 u^{2}-u^{3}\right)+p_{1}\left(3 u-6 u^{2}+3 u^{3}\right)+p_{2}\left(3 u^{2}-3 u^{3}\right)+p_{3}\left(u^{3}\right)
$$

where:

$\mathrm{p}$ : is the control point of the polygon

$\mathrm{u}$ : parameter $0-1$

This is a brief approach to understanding how to model a free form curve. There are many other equations to create a Spline curve by computer, like NURBS (non-uniform rational Bspline) and others. A 3D surface is created analogous to a curve, but using one more axes. 


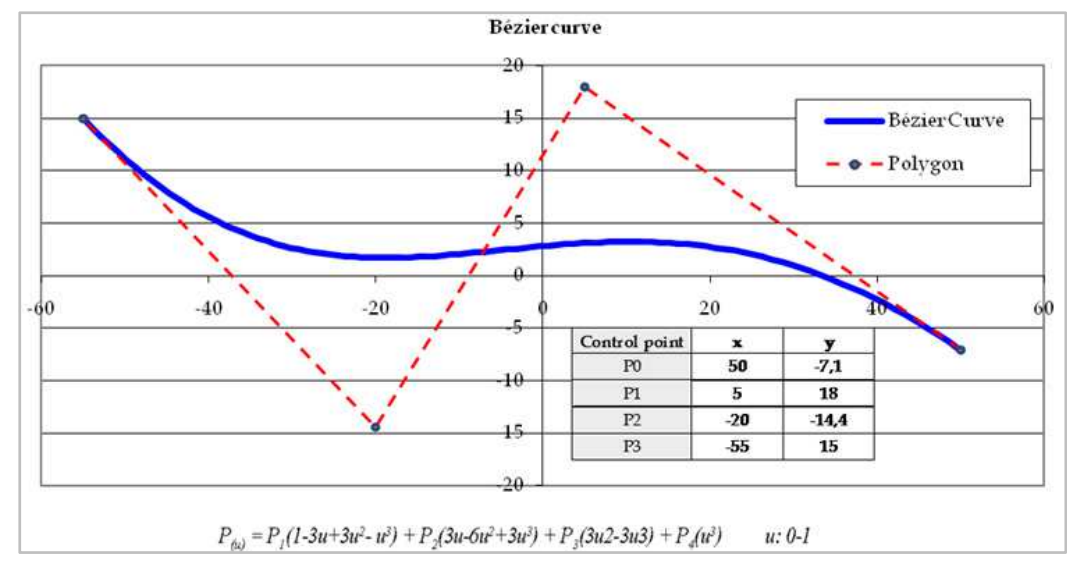

Fig. 2. A Bézier curve plotted in graphic software

It is also important to distinguish that free form geometries do not have to be associated with complex products. Many complex products have no complex shapes, for instance, a machine centre. It has many components, high precision assembling, and relatively high cost. However, in general, its components have prismatic or rotation shapes. Instead a simple bottle of pop soda has no assembly, it does not require such precision, and it is not an expensive product. However its shape are quite complex, and most of the time to manufacture complex geometries are much more expensive than simple forms, once it required high level of technology, like CAx.

\section{Computer aided free form manufacturing}

Due to its complexity, free form shapes cannot be machined by a conventional machines driven by human. It requires a CNC machine and the free form tool paths have to be calculated by software. After machined the geometry, geometric errors have to be accessed. Once again, due to its complexity it is not possible to make any inspection by ordinary metrology devices. Therefore, such cases require a measure machine coordinate (or any order technique to accessed geometric errors, like as laser or photometry).

So, the manufacturing steps of complex forms requires different CAx systems. Within these systems the following stand out:

- $\quad$ CAD (Computer Aided Design/Drafting). Software to aid the Project, design, mould industrially design the products.

- $\quad$ CAM (Computer Aided Manufacturing). Software to aid manufacturing activities.

- CAI (Computer Aided Inspection). Software to aid the inspection of geometric forms.

- CAE (Computer Aided Engineering). Software to aid the simulation of mechanical, strength, temperature, pressure etc.

The Figure 3 presents the integration of some CAx systems required to manufacture for free form geometries. 
Besides, CAE (Computer Aided Engineering) software can be used to simulate the product mechanical requirements or the manufacturing process. However, in most of the times it is not essential for free form manufacturing such that mentioned above. You can find a detailed description about the CAx for free form modelling and manufacturing ahead in this chapter.

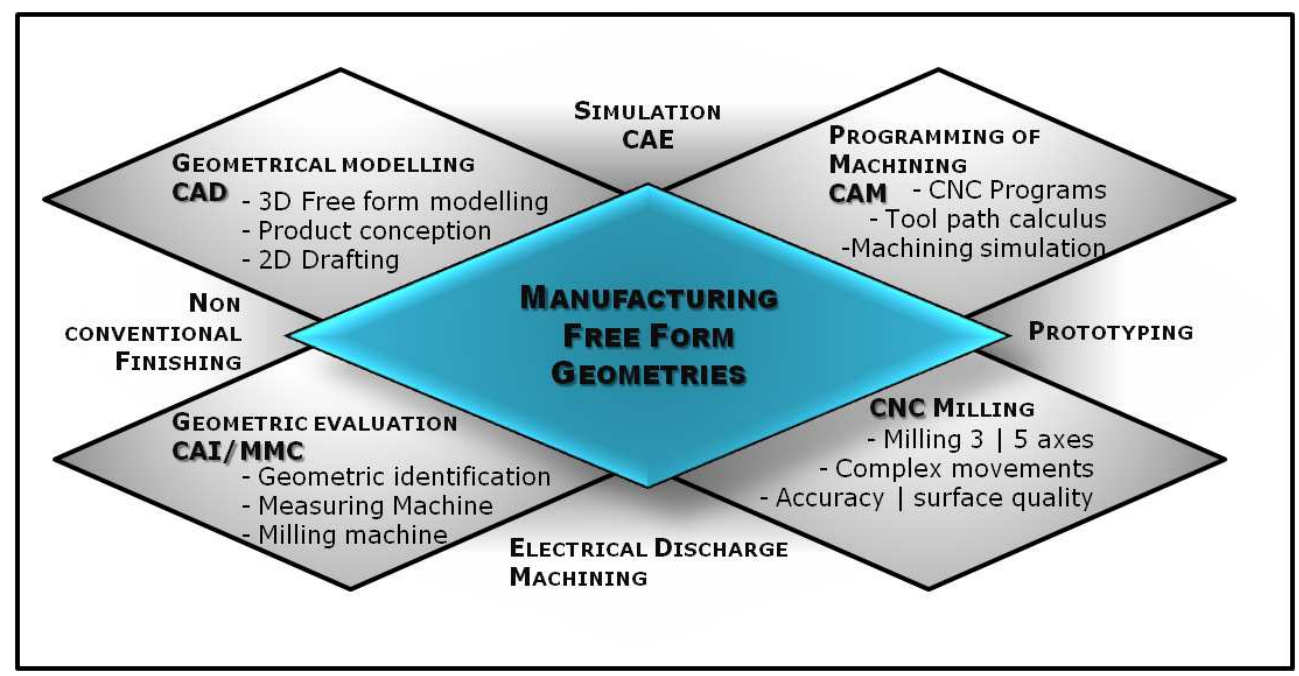

Fig. 3. Some CAx application on free form manufacturing

\section{Main concerns about the manufacturing process of free form geometries}

In the contemporary world the manufacture of free form geometries is carried out first by milling and electrical discharge machining (EDM) when necessary. Newer manufacturing process has been developed such as fabrication by deposition, like as the rapid prototyping, but yet the first both process are still the mainly ones and they are taken into account in this chapter.

Usually, milling is the first option due to the inconveniences of the EDM processes, because EDM it requires an extra effort to modelling the electrodes, CNC programming and milling the electrodes, time consumption for EDM machining itself, besides the EDM can injure the machined surface integrity. The EDM process is used where the milling tool cannot reach due to geometry limitation, or it may be required in specific cases. The 5 axis milling is a drive force for manufacturing free form shapes, once that the cost are reducing and the technology is getting raising.

For milling complex forms the generation of CNC programs should be fulfilled by a CAM system. The user should initially have a geometric model of CAD 3D, which should be used by CAM as a base to calculate the tool path. After the calculation the route of the tool should be simulated and subsequently coded in commands, which should be interpreted and transformed by the $\mathrm{CNC}$ machine in movements and actions. The Figure 4 illustrates the procedure to manufacture a free for geometry by CAM software. 


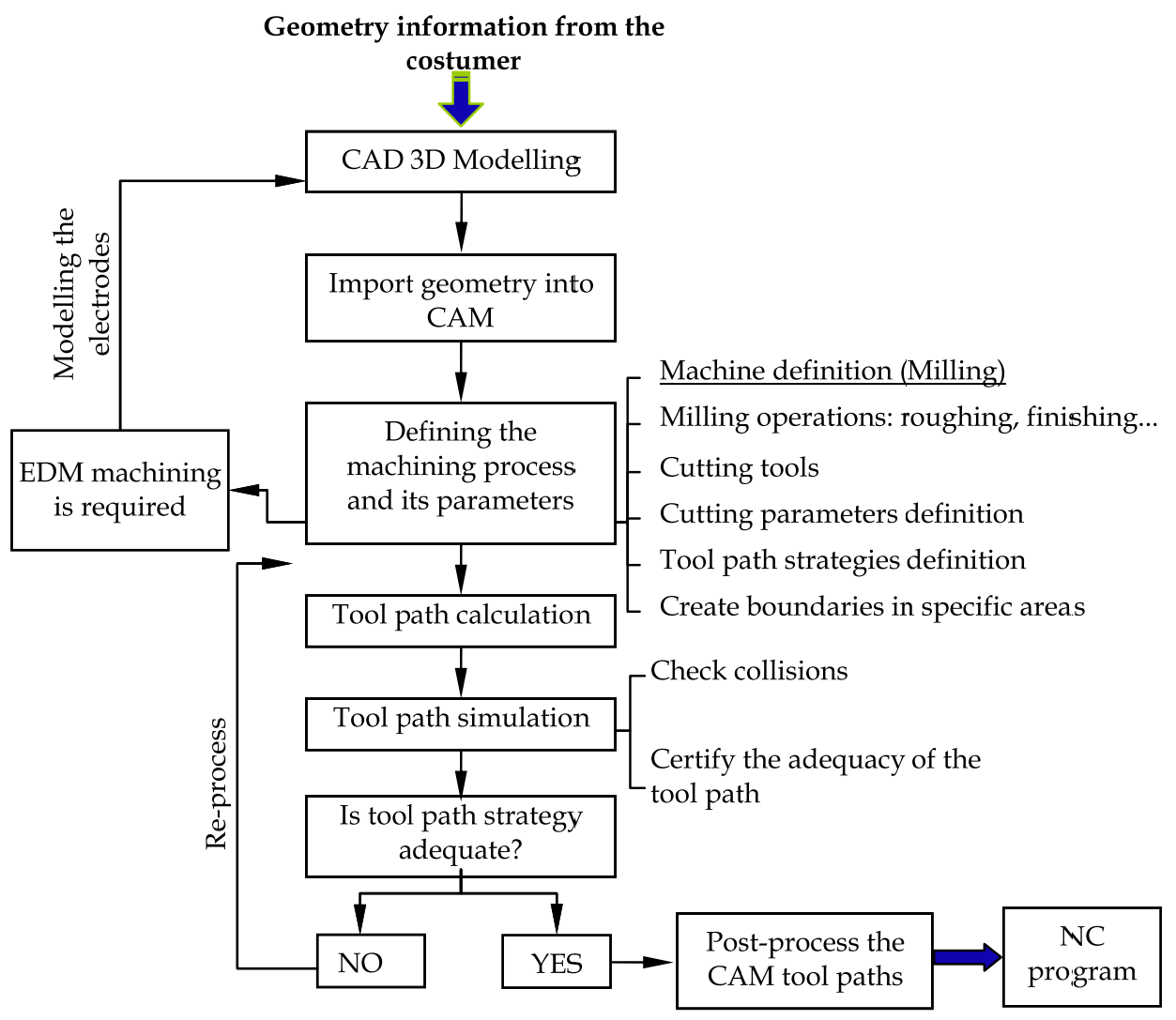

Fig. 4. Procedure to manufacturing free form geometries by CAM software

The mainly features of free form milling can be listed as following.

\subsection{Roughing milling in free form geometry manufacturing}

This operation can happen in two circumstances:

i. The raw material is furnished in a prismatic format. The software needs this information to calculate the extensions of the path calculated, the height, the width and the length. The geometric block, when prismatic, should be defined easily with the CAM software.

ii. The geometry of the raw material is furnished in specific format. It could be manufactured before, by foundry, or any other processes. Like this the prime material for milling should have geometry close to the final form. (Fig. 5b). In these cases the user should model the geometry form of the prime material using a CAD software. This geometry must be exported to CAM to recognize the geometry as being the block of raw material to be milled. In this way CAM calculates the path just between the block of prime material and the material being milled. This avoids unnecessary movements between the block and the tool, which economizes milling time. 


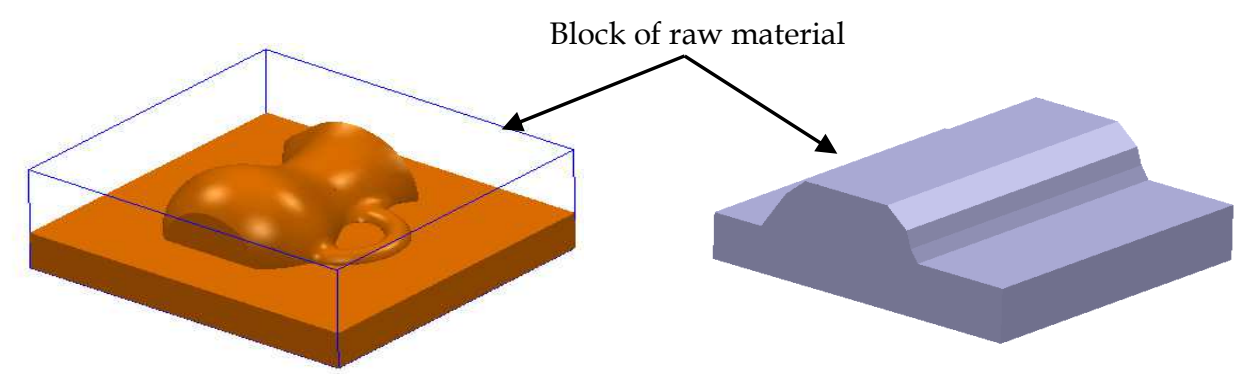

(a) Row material cubic

(b) Row material near net shape

Fig. 5. Blank material options

The roughing employs movements in $2 \frac{1}{2}$ axis. The tool is positioned at a determined height in $\mathrm{Z}$. After movements are executed in 2 axes for the removal of material at this level. When the removal of material at this level is finalized the tool is then positioned at a new level of $Z$ (defined by the depth of cut), the material is removed at this plan. The process is repeated until the roughing is complete. End-mill with corner radius is the cutting tool frequently used for this operation.

\subsection{Stock of material for finishing operations (stock material)}

Normally some stock of material (also called thickness) is left for the finishing operation. The value of this stock of material is set in the CAM by the user, and it is an offset of the geometry to be machined. For steels machining an average of values that are employed are between 0.5 $\mathrm{mm}$ and $1 \mathrm{~mm}$ of stock that should be removed in the finishing operation. One value given to the thickness of the leftover of metal could represent a significant effort in finishing and this could prejudice the quality of the surface and also prejudice two situations: a) geometric errors caused by deflection of the tool; b) elevated surface roughness. Low stock can also prejudice the quality of the surface therefore tool can come across difficulties when carrying out properly, the material being cut. This causes passing and crushing material that should be cut. Once again each case ought to be methodically evaluated.

When milling complex shapes besides this theoretical stock, a volume of material cannot be removed from the roughing, due to topological limitations between the surface complexity and cutting tool geometry.

An extra volume of stock can be found on the degrees of material formed after roughing as illustrated in Figure 6(a). This extra volume depends of the depth of cut, the curvature of the surface and the geometry of the tool. Cutting tools with an extreme radius or spherical tools minimize but do not eliminate this condition.

To optimize roughing, tools with greater diameters are employed. In regions of corners, many times the cutting tool is limited to reach and to carry out the milling. Then again an extra volume of material is left by the roughing (Figure 6). 


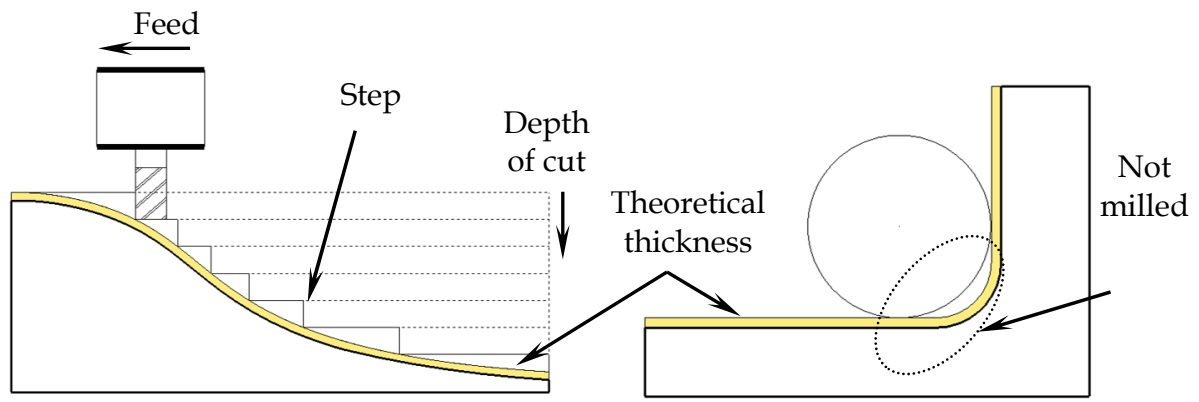

Fig. 6. Thickness to finishing

Frequently the differences of the volume of material beyond the theoretical stock is not cautiously evaluated, which has repercussions with possible problems in the milling. It is common that the machinists of the $\mathrm{CNC}$ machines reduce the speed during the milling process manually in some regions of the piece. To minimize this problem of semi-finishing operations have the objective to remove this excess of material and maintaining the stock as close as possible to the theory value (offset of geometry).

\subsection{The tool-surface contact in free form milling}

Finishing operation of machining can be considered the most important along the manufacturing free form shapes, once that implicates directly on the final product, in terms of surface integrity and geometric errors. A ball-end cutting tool is the most suitable to finish free form shapes by milling due to this geometry which permits to reach different curvature shapes. However, this tool implicates in specifics characteristics in milling free form shapes, once that the contact tool/surface alternates constantly and affects directly the milling process, in terms of cutting speed, cutting force, surface quality and so on (see also SOUZA et al, 2010).

First, the cutting speed usually cannot be calculated by nominal tool diameter. It has to be taken into account the effective diameter according to the axial cutting depth together to shape curvature. For a planar surface milled by a ball-end tool (Figure 7), the maximum effective diameter can be calculated by equation 2 . But it has to be taken into account the radius of curvature of a free form geometry alternates constantly (Figure $7 \mathrm{~b}$ ). Therefore, the effective tool diameter following this oscillation and thus, the cutting speed as well. This is one of the drawbacks because the machining process became unstable.

$$
R_{e f}=\sqrt{R^{2}-(R-a p)^{2}}
$$

Where:

Ref $=$ Effective radius $[\mathrm{mm}]$

$a p=$ axial depth of cutting $[\mathrm{mm}]$

$R=$ Tool radius $[\mathrm{mm}]$ 


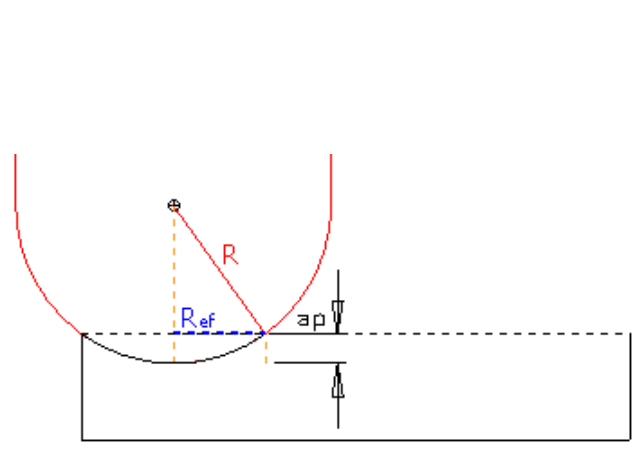

(a) effective radius

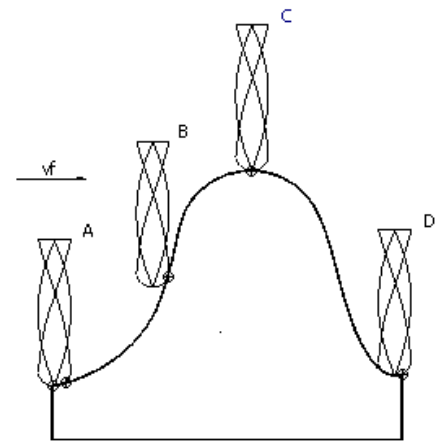

(b) contact tool-surface

Fig. 7. Effective tool diameter and contact tool-surface in free form milling

$$
R_{e f}=\sqrt{R^{2}-(R-a p)^{2}}
$$

Where:

Ref $=$ Effective radius $[\mathrm{mm}]$

$a p=$ axial depth of cutting $[\mathrm{mm}]$

$R=$ Tool radius $[\mathrm{mm}]$

Another weakness of this process is the surface roughness. First, the cutting speed is zero in the extreme centre of the cutting tool. Therefore, when the centre of the tool is in the cutting area, the tool smash and do not cut the material. This phenomenon affects negatively the surface roughness. The ball mill also leaves a material remain between the cutting passes, known as cusp high. The cusp high depends upon the axial (ap) and radial depth per cut (ae), tool diameter and surface curvature (see Figure 8).

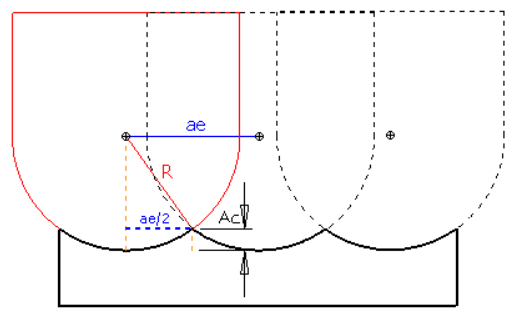

Fig. 8. Cusp high in a planar surface and contact tool-surface in free form milling

Where:

$\mathrm{Ac}=\operatorname{cup}$ high $[\mathrm{mm}]$

ae $=$ radial depth of cut $[\mathrm{mm}]$

$\mathrm{R}=$ tool radius $[\mathrm{mm}]$ 


\subsection{Machine limits}

High frequency spindles, in combination with high feed rates are a more precise characterization for HSC (High Speed Cutting), especially for die and mould manufacturing. Literature mention HSC operations using feed rates as high as $20.000 \mathrm{~mm} / \mathrm{min}$. However, the feed rate varies severely along the path. These feed rate oscillations depend on two limitations: mechanical (inertia) and electronic (CNC control). In the first case, the machine does not achieve acceleration and deceleration as fast as required by a specific programmed feed rate. In the second, CNC limits the feed rate to a maximum value according to its processing capacity, considering a tool path is described by small linear segments. Feed rate oscillation happens even in the most updated CNC machines and it drastically affects machining time and surface quality. The ordinary CAM software estimates the machining time considering that the feed rate is kept constant as set in the NC program; the software does not consider the CNC and machine's limitations. Therefore, the machining time estimated by CAM becomes very inaccurate [COELHO et al, 2010].

\subsubsection{Feed rate oscillation in free form milling}

A free form tool path is described by an small straight line interpolation. The respective CNC program generated by a CAM software create an huge NC programs and it represents a great amount of information for the $\mathrm{CNC}$ machine to process in short periods of time. As a result, the $\mathrm{CNC}$ machine may have to reduce its feed rate to manage such large amounts of information. Therefore, the linear segment length is directly related to the feed rate during milling and, as a consequence, to the machining time. Better description about the topic can be found ahead in this chapter.

\subsubsection{Machine control}

A typical CNC control of a machine tool has two major responsibilities: i) the servo control loop and ii) the interpolator, responsible for coordinating the machine tool axis motions. The NC program, as well as others error compensation programs and routines, is located in a higher level of the control hierarchy. Supervisory tasks such as final part measurements and corrections can be accomplished by the CNC getting feedback from other controllers.

From the machine point of view, acceleration, deceleration and kinematics characteristics could be the feed limits in a machining process. According to ELBESTAWI (1997), the main issues in high speed servo control are feed rate planning and servo control loop laws. The users and/or the machine tool builders have to choose between path error and feed rate error. If the path error is allowed, the machine tries to run at the programmed feed rate; however, the trajectory is not guaranteed. If the path error has to be low, the machine keeps the path accuracy, but the feed rate can be lower than the programmed one.

In free form milling, the linear interpolation of straight line segments means a high volume of information to be processed by the CNC. Therefore, in order to manage the tasks to be carried out in real time during machining movements (i.e.: machine control loop, jerk limitation, interpolation, and so on), the $\mathrm{CNC}$ reduces the feed rate according to its capacity. 
a. Block Processing Time. To run the machine in linear path, the points of the NC program have to be interpolated to obtain the movement in one or more machine axis. This task is done by the CNC interpolator. The interpolator has to calculate the speed of each servomotor of the machine tool in every block. The real-time interpolator converts the CL path to the motion trajectories of the orthogonal machine axes so as to coordinate their motion in three-axis surface machining. This task is known as CNC block processing time. The main CNC tasks are presented as follows:

b. Control loop. In addition to the interpolation, the current CNC controller also has to check feedback devices in order to maintain the machine's high precision. The machine closes a control loop using devices such as encoders and linear scales. In the CNC machine system, the practical tool location is measured by the feedback devices at a specific time, i.e., sampling interval. The sampling interval is about 0.001 seconds, depending on the CNC milling machine; this represents another task to be calculated by the CNC.

c. Motion algorithms. Other algorithms developed to aid machine motion and positioning, such as look-ahead, feed forward control, and jerk limitation are also implemented in present CNC machine. Therefore, all the tasks mentioned above have to be done by the CNC in a very short time.

When machining complex geometries, the machine often encounters curved surfaces, where short segments are required to closely follow the profile. When that occurs with the NC program set with high feed rates the machine slows down and sometimes oscillates imitating a vibration behaviour.

Feed rate oscillation happens even in the most updated CNC machines and depends on servo response, feedback devices, CNC characteristics and its implementation on the machine tool.

\subsection{Tool paths described by polynomial spline}

The polynomial Spline curve can also be used to describe complex tool paths (SOUZA et al, 2010). Therefore the NC program generated will not contain the traditional commands (G01, G02 or G03), but there is a new syntax which illustrates the tool path and the polygon control which defines the curve. The Cartesian points are used to define the polygon. There are different syntaxes for the entrance of the spline data. This variation agrees with the numerical commands used. Below can be found an example of syntax for Spline movement which is generated in accordance with nomenclature of CNC FANUC.

For the generation of CNC programs using polynomials Spline, the CAM systems permit this resource which do not generate directly. The CNC programs contain Spline functions that are generated leaving a CNC linear program in straight segments. With this initial tolerance is used to calculate the linear program adding the tolerance calculation of the Spline program. For the process to be adequate the user should scientifically plan the most efficient way to work with these tolerances. Bibliographical research done during the development of this work has found very practical analysis.

YAU e KUO (2001) developed an application that converted traditional CNC generated programs for interpolation linear in CNC programs with Spline format. This method of 
work has implemented sophisticated numerical commands. In this case the data entered into a CNC program containing commands G01 and internally the $\mathrm{CNC}$ converts the straight paths into Spline equations, proportioning better performance than the traditional method of straight segments, however this conversion must be attributed to another value of tolerance. A deeper understanding can be found in SOUZA (2004) and SOUZA \& COELHO (2007) $)^{\mathrm{a}, \mathrm{b}}$.

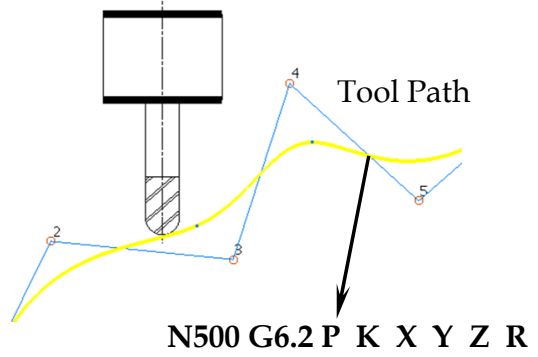

Where:

G6.2 - syntax of NURBS

$[\mathrm{P}]$ - degree of the polynomial equation

$[\mathrm{K}]$ - vector on the control point (knot)

$[\mathrm{X}, \mathrm{Y}, \mathrm{Z}]$ - coordinate of the control points

$[R]$ - weight on the control points (weight)

Fig. 9. Spline program line for the FANUC command

\section{CAM systems (Computer Aided Manufacturing)}

In the milling of complex forms, due to complexity of the paths of tool, the generation of the programs for CNC machines should be deeds with aid of software, the CAM (Computer Aided Manufacturing). Although the acronym CAM expresses a generic system for support a chain of manufacture, the term CAM is commonly employed for the generation of programs for CNC machines.

The CAM calculates the paths of the tool for carry out milling, besides simulating the movement of the machine for certification, and generates the $\mathrm{CNC}$ programs for the manufacturing of the piece.

At present, the biggest application of CAM software is for the programming of machining by milling, in special 3 to 5 simultaneous axes. CAM software can also generates CNC commands for EDM machines, lathes, and laser or water pressure cutting machines, among other applications carried out by equipment controlled by a $\mathrm{CNC}$.

The CAM systems can be independent software that works in assembly with any application $\mathrm{CAD}$, in general $3 \mathrm{D}$, needing this, the importing of geometries in formatstandard, such as, IGES, STL, VDA-FS, etc.

A CAM system can be used to develop three main activities: i) calculate the tool paths for the machining the $\mathrm{CNC}$ machine; ii) carry out the simulation of the machining calculated; 
iii) generate programs for specific CNC machines. Doubtless the first function is more important and can represent the main difference between the diverse CAM systems available in the present market.

The operations of milling, focusing on the making of complex forms, can be classified as: roughing, semi-finishing, relief of corners and finishing. For each one of these operations exist different techniques and peculiarities of programming with CAM.

\subsection{Tool paths for roughing}

Upon carrying out machining by milling of a surface, the tool can traverse different routes. The CAM systems offer different tool path options. Although any one of these paths can carry out the operation of machining, they can have repercussions in the trial, differentiating itself mainly regarding the time of machining, demands of the tool of cut, and superficial quality. The identification of these options should be carried out by the user. The CAM systems presented are not able to identify the best strategies of machining for specific cases, according to geometry it be machined, mainly in complex forms.

For the operations of roughing for milling, besides the paths of parallels passes, the CAM systems still developed other strategies as: paths in offset, paths based in the internal contour of the geometry, paths based on the external contour of the geometry.

In the roughing operations, the dimensions of the block of raw material can be utilized to limit the tool paths calculated by the CAM. In this way, this resource permits the user create machining paths in a specific region of the piece.

\subsection{Programming operations of semi-finishing}

Although there is not a standard definition of semi-finishing for this application, here it will be naming semi-finishing the milling operations that has for objective become constant the value of the stock of material to be milled after by the finishing operation.

For this reason, the regions with remaining material after roughing besides the theoretical stock should be machined. In general, the semi-finishing is an operation of "roughing" in $2 \frac{1 / 2}{2}$ axes, in specific regions and can be divided in two phases: a) operations for removal of the degrees and b) operations for relief of corners, as presented previously.

\section{Programming operations for the removal of degrees}

This algorithm, available in some CAM systems, identifies automatically the steps left by the operation of roughing, considering the axial depth of cut and the geometry of the tool, in function of the curvature of the machined surface.

Some systems create a model with the geometry of the piece after roughing, with all the excess of material. This geometry then is utilized like a block of raw material for the semifinishing (Figure 10).

\section{Programming operations for the relief of corners}

This operation has the purpose of remove the material not machined in roughing, in areas not reached by the cutting tool, due to relatively big diameter tools used (tools characteristics of the operations of roughing), as presented previously. 


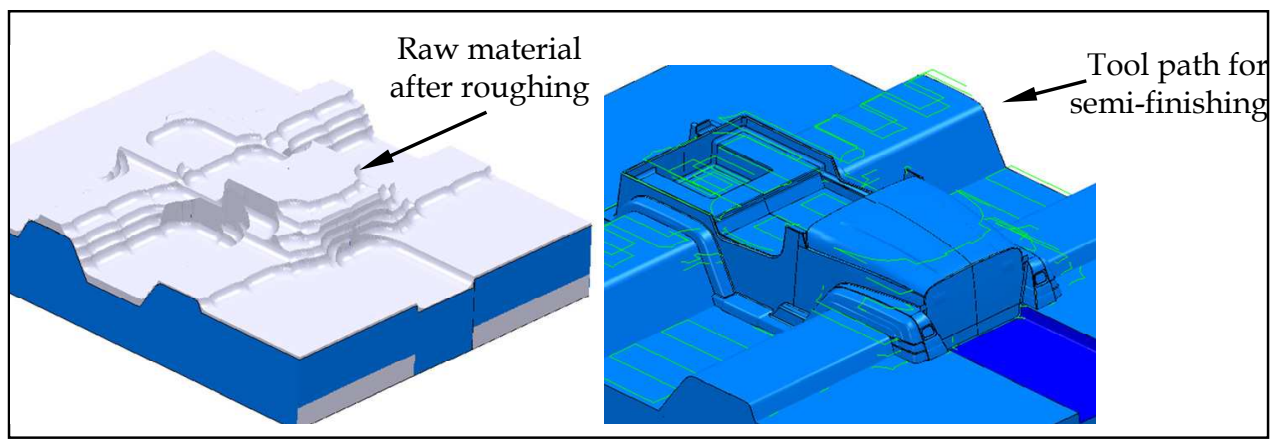

Fig. 10. A methodology to define the block of raw material for the semi-finishing

Therefore, in the corners relief operation should be employ tools with diameters smaller than the tools utilized in roughing.

The more modern CAM software presents a tool for identifying these regions automatically. The algorithm identifies the corners of the geometry of the piece where it has a radius less than the ray of the tool of thins. In the finishing of this algorithm the software requests the information of the diameter of the tool that was employed in roughing, identifies the areas and calculates the machine paths of these regions, employing a tool of smaller diameter.

\subsection{Programming finishing operations}

This is an operation of three-dimensional milling, utilizing 3, 4, or 5 free axes, according to arrangement of the machine-tool. This operation is going to remove all excess material of the previous operations and finalize the operations of milling.

In the finish, the axial cut depth parameter passes the tolerance value and depending on the position of the tool-piece, this depth will not be necessarily axial, as illustrated the Figure 11. The tool parameter of geometrical cut itself maintains the same concept.

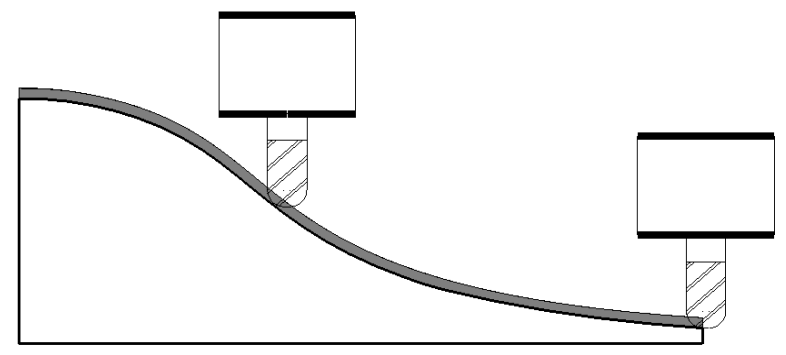

Fig. 11. Finishing depth of cut

The CAM systems present offer different algorithms to calculate machine paths for finishing. The main principles are:

a. Parallel passes horizontally constant. Probably this is one of the first algorithms for finish path calculation to be developed, and is widely employed. Known as 
strategy in Zig-Zag or Raster. The software previously creates the parallel paths in a horizontal parametric plan, superior to the geometry it be machined, considering the value of the radial pass informed by the user. These paths, which are spaced out equally in this plan, are projected about the surface that will be machined in the finish, as illustrated it Figure 12.

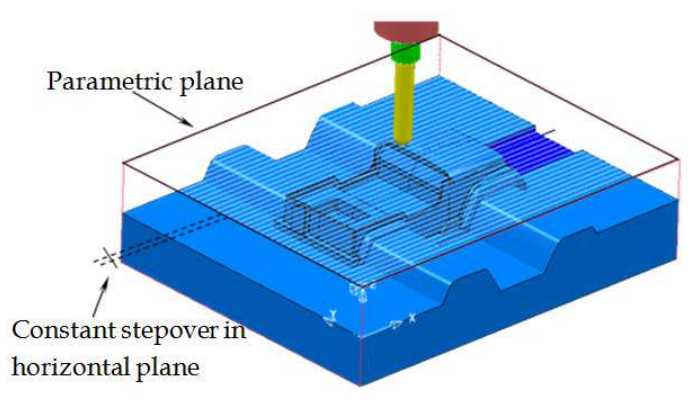

(a) Preview of tool path calculus on the parametric plane

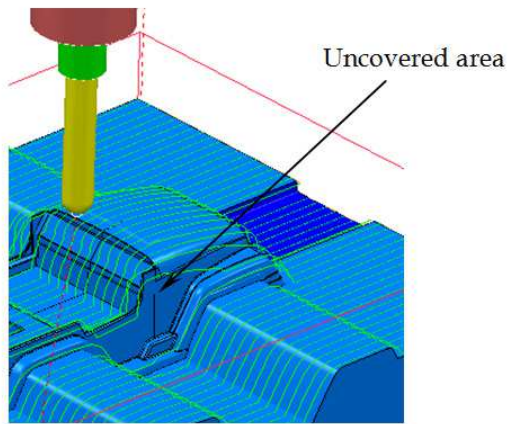

(b) Calculated tool paths on the surface

Fig. 12. Finishing milling by parallel tool paths strategy

It should be noticed that this strategy is adequate for horizontal regions of the geometry, where the distances between the passes from the tool remain constant, as in the plan parametric. In the regions where the inclination is vertical, the tool passes stay distant, having repercussions in areas that will not be machined in the finish. For motivate, the combination of different strategies in machining, the same piece, is in many ways necessary and convenient.

b. Parallel passes vertically constant. This strategy is equivalent to previous, considering now the vertical sense. Therefore, the vertical areas of the geometry will be more beneficiary than the horizontal areas. Many times, the combination of both the strategies are employed.

c. Passes in three-dimensional offset. In this algorithm the paths of the tool are calculated by offset of the contour of the geometry it be machined, maintaining the pass constant even in complex geometries. The inconvenient one of these options of path is the marks in the surface left by the tool in the transition between the passes.

d. Spiral passes conforming to three dimensional profiles. There are more sophisticated algorithms, which calculate the path of the tool in spiral, following the contour of the geometry. Few systems CAM has implemented this algorithm successfully.

e. Passes for finishing corners. These algorithms identify corners of the geometry it will be ended up a tool of small diameter.

The CAM systems offer an amount of finishing strategies. The user should identify the adequate for each case.

The choice among the different paths of tool can represent a big influence about the necessary time for the machining, cutting tool load and superficial quality. This it is aggravated in the machining of complex surfaces, where the quality of the trial is not 
influenced barely by the equal tool/piece, as is the case of the milling common, but depends strongly of the adequate utilization of the strategy of cut for a determined rank of curvature of the surface and to its association with the adjacent surfaces.

\subsection{Borders of machining for finishing in specific areas}

Similar upon roughing, the operations of finish also should possess limitations of the areas of machining. These borders of finish (boundaries) can be created by different ways:

- Selection of faces of the piece. Upon selecting a face of the geometry, the CAM can create a border for carry out a specific finish.

- Manual creation. The user designs, in a plan 2D, the region that is going to limit to machining, that can be in the inside of the border or external, in agreement selection of the user.

- Geometry originating from the CAD. Many times, the user has the option of extract a curve of the geometry, utilizing the CAD, and export it for the CAM, transforming it in border of machining.

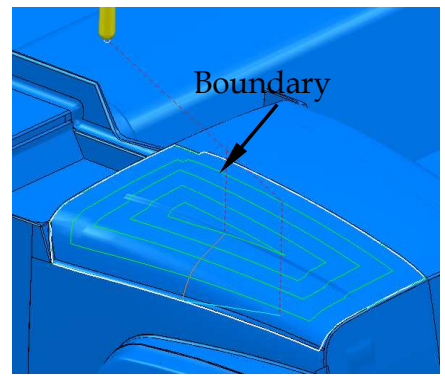

(a) Boundary calculated by an specific surface

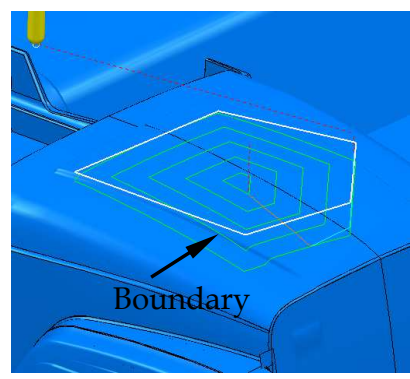

(b) Boundary created by sketch wireframe

Fig. 13. Boundary for finishing

There are other ways to calculate boundaries, in agreement the software CAM utilized. In many cases, the software CAM utilizes the geometry of the boundary as information help for carry out the calculations of the paths of the tool. Boundaries improperly formed can impact the quality of the machining process. It must itself consider that this boundary many times is a curve kind Spline. Inflection in this curves, which will transformed in a boundary machining are transferred for the tool paths, injuring the quality of machining. A help with the $\mathrm{CAD}$ in these occasions can be convenient, in agreement demand of the agreement.

\subsection{Simulation of the machining in the CAM software}

The phase of simulation can possess different objectives:

a. Verify undesired tool invasions into the part (gouge). These invasions can occur either due to error during tool path calculation (software) or by misconceptions of the user, in the definitions of the movements and specific settings. 
b. Verify tool path adequacy after calculation. Due to the big cut strategies range, associated to the diversity of geometrical forms, even for experienced users, in many cases, barely is possible estimate the quality and adaptation of the path calculated, through the simulation.

c. Verify tool path collisions according to tool holder geometry. The software should consider the tool balancing length, his diameter and the geometrical characteristics of the tool holder.

Advanced CAM, beyond identify the areas of possible collisions, the software can identifies the maximum depth that the system tool/tool holder can reach without collisions; divides the path in a first one breaks (that will be the first program $\mathrm{CNC}$ ), as far as the system can achieve, and for the near depth (second program CNC), the CAM identifies the length of necessary. In this way, the initial path, with a smaller length should be more stable, as regards vibrations, and by that, can be quicker. Already in the second programs the user should have bigger caution in the machining. This can be an important aid in the CNC programming by the software CAM.

d. Simulate collisions with the fixation of the piece on the table of the machine. Diverse CAM systems are going to attribute geometrical forms, many times modelled in the $\mathrm{CAD}$, for represent the systems of fixation of the piece on the table of the machine, when necessary. With that, the CAM passes it calculates the paths avoiding of the geometries of fixation.

e. Simulation of machining $\mathbf{5}$ axes. Due to the complexity of movements, with 5 ranks of liberty, the simulation of 5-axis machining is of vital importance for movable and fixed parts of the machine does not collide. In that case, is very important the CAM carry out the simulation employing all the geometry of the machine, limits of movement, and fixations of the piece. The Figure 14 illustrates a machining 5 axes.

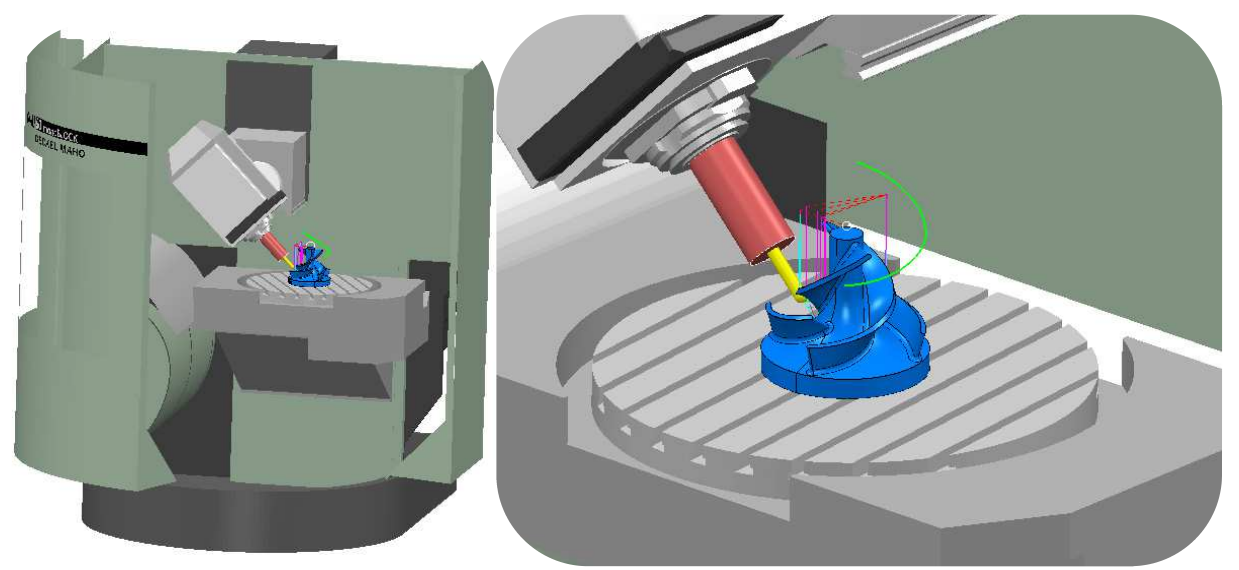

Fig. 14. Simulation of CNC machining considering the machine

Due to complexity of the milling 5 axes, the simulation CAM can take a significant time in this manufacturing process.

It is important to note that in all these simulation steps presented above are used to simulate the tool paths in the computational format of the software CAM. These paths do not be still 
the program $\mathrm{CNC}$ that will go for the machine. The program $\mathrm{CNC}$ is created starting from the paths, in a phase named post-processing, detailed it follow. Mainly in the machining 5 axes, errors can occur in the post-processing. A simulation carried out in the CAM cannot correspond with some movements of the machine, being able to cause collisions. Specific software for simulation exists of the program $\mathrm{CNC}$ and of the machine. This simulation confers bigger security to the trial.

\subsection{An introduction to tool path calculation for free form geometries}

The calculation of the tool path is the main function of the software CAM. The result of the final part is straightly related with the quality of the tool path, implying in the quality of the surface machined, dimensional errors and times of path calculation and real machining. The algorithms and the methodologies for tool path calculation are developed by each software house, and represent industrial secrets.

In the current procedure used for most commercial CAM codes to generate free form tool paths, the system first calculates the cutter-contact points (CC) over the 3D CAD geometry. Then the tool offset is included resulting in the cutter-location (CL) points, which are used to generate the NC program blocks; using the traditional method to create a free-form tool path, i.e., the linear interpolation of straight-line segments, with continuity $\mathrm{C}^{0}$. These $\mathrm{CL}$ points are easily transformed into the ordinary G01 CNC code, according to DIN 66025.

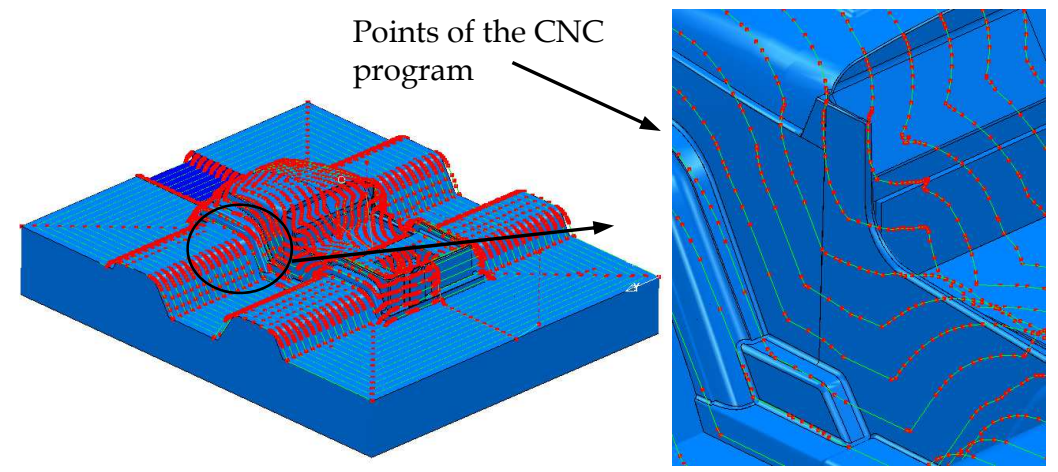

Fig. 15. Points that describe a complex tool path for milling

The length of these segments is related with the tolerances of calculation in the CAM and the degree of curvature of the surface. The CAM software adjusts the tool path segment lengths to fit inside a tolerance band defined by the user, known as "chord error". The smaller the tolerance band, the closer the tool path will be it to you the original CAD 3D model. The smaller the tolerance band, the closer the tool path will be to the original CAD 3D model. Thus linear segments are generated by such a procedure and, for highly curved paths, the number of segments increases dramatically, along with the CNC program size. The Figure 16 shows the influence of the curvature of the surface in the length of the segments of straight lines of a program $\mathrm{CNC}$, calculated inside a same band of tolerance.

Some CAM systems allow to establish through a band of variation, limits of tolerances with the possibility of deviation from the path inward and/or for outward of the geometry. The 
lower the tolerance band, the closer will be the tool paths from the surface to be machined, however, smaller will be the followings segments of lines, CNC programs will possess greater volume of data, since more lines comprise the program and this characteristic will be able to limit the feed rate during machining, particularly in complex forms. The machine reduces the programmed feed rate, as studied by SOUZA \& COELHO (2007).

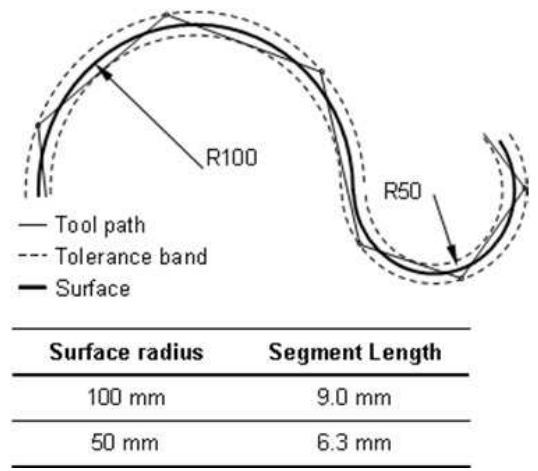

Fig. 16. Tolerance to calculate the CNC programs

The path of the tool calculated by a system CAM represents the central extremity of the tool, and by that, the path is not a simple offset of the surface it be machined. To develop the calculation, the software should identify two positions: a) Point of contact of the tool with the surface, known as CC - Cutter Contact and b) the point that represents the path of the tool (its central extremity), named of CL - Cutter Location.

The lines of the programs $\mathrm{CNC}$, referring the paths of the tool, contain the Cartesian information of each points CL. It must be noticed that the Cutter Contact is the point which should be inside this band of tolerance and not the path of the tool or the Cutter Location.

Deferential ways exist to calculate both, the CC and CL. These algorithms are virtues of a CAM, therefore can have repercussions in the time of calculation, time of machining and quality of the surface machined. Initially, in a certain moment, and then it should calculate the final point the tool centre (CL) points that are linearly interpolated to generate the tool paths.

An approach for calculate the $\mathrm{CC}$ in polynomial surfaces is the division of the own polygon of control of that describes the curve or surface. This subdivision can identify points about the curve or surfaces, which can be the CC. Others approaches are developed for such end.

After it identified the CC, the CL must be calculated. The Figure 17 exemplifies a possibility to calculate the $\mathrm{CL}$ for two points of a path, considering that a previous algorithm already have found the CC. In this example, knowing to normal of the surface in the point desired (CC) and angle that this normal is found regarding the axis of the coordinates, is able to the CL be found through the ray of the tool.

\subsection{Circular and linear tool path interpolation}

Due to the inconveniences of the traditional linear interpolation method for machining complex forms, an alternative is to use circular interpolation associated with linear 
interpolation to describe a tool path. That combination can maintain the continuity of the curve $\mathrm{C}^{1}$ and showed many advantages about the isolated utilization of segments of straight line (SOUZA, 2004). This approach, however, can only be applied to planar motion by interpolating two axes of the CNC machine, since the great majority of CAD / CAM / CNC are unable to carry out circular interpolation in three dimensions. Thus, it has aimed utilize polynomials Spline to describe complex tool paths.

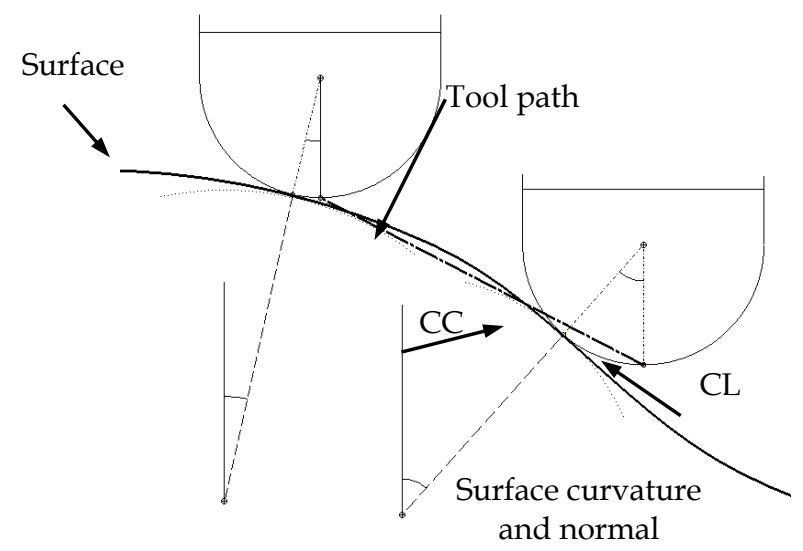

Fig. 17. Determination of CL by CC

\subsection{Post-process task}

After the CAM calculates and simulates the tool paths, there is the phase of post-processing, which is responsible for transforming these paths into CNC programs.

The initial calculation in the system CAM generates a generic file, without specifications. This file is known like Cutter Location File (CLF).

The post-processing has the function of encode the paths calculated by any software CAM in specific commands for a given configuration machine/CNC.

Therefore, two elements are necessary for create the program CNC:

- Tool path calculated by the CAM in computational format (CLF).

- Post-processor - computational program that reads the file CLF and converts it into a program to be performed in a given combination of machine/CNC.

For each equipment, machine/CNC should be developed a specific post-processor. The procedure for the creation of post-processors and development of programs CNC is presented in the Figure 18.

The information of the machine is related to its physical characteristics, as maxim movement of the axes, number of axes, speeds etc. Regarding the numerical control, there is a wide range of $\mathrm{CNCs}$ on the market today, ISO 66025 standardizes the main functions codes CNC. There are several distinctions among suppliers. The simplest cases involve, as example, the utilization of point or comma for separate decimal places; number of decimal places demanded; block end character need; command for beginning and end of program, among others. 
In general, the post-processor is provided with the CAM software, and developed according to user requirements, and the CNC machine.

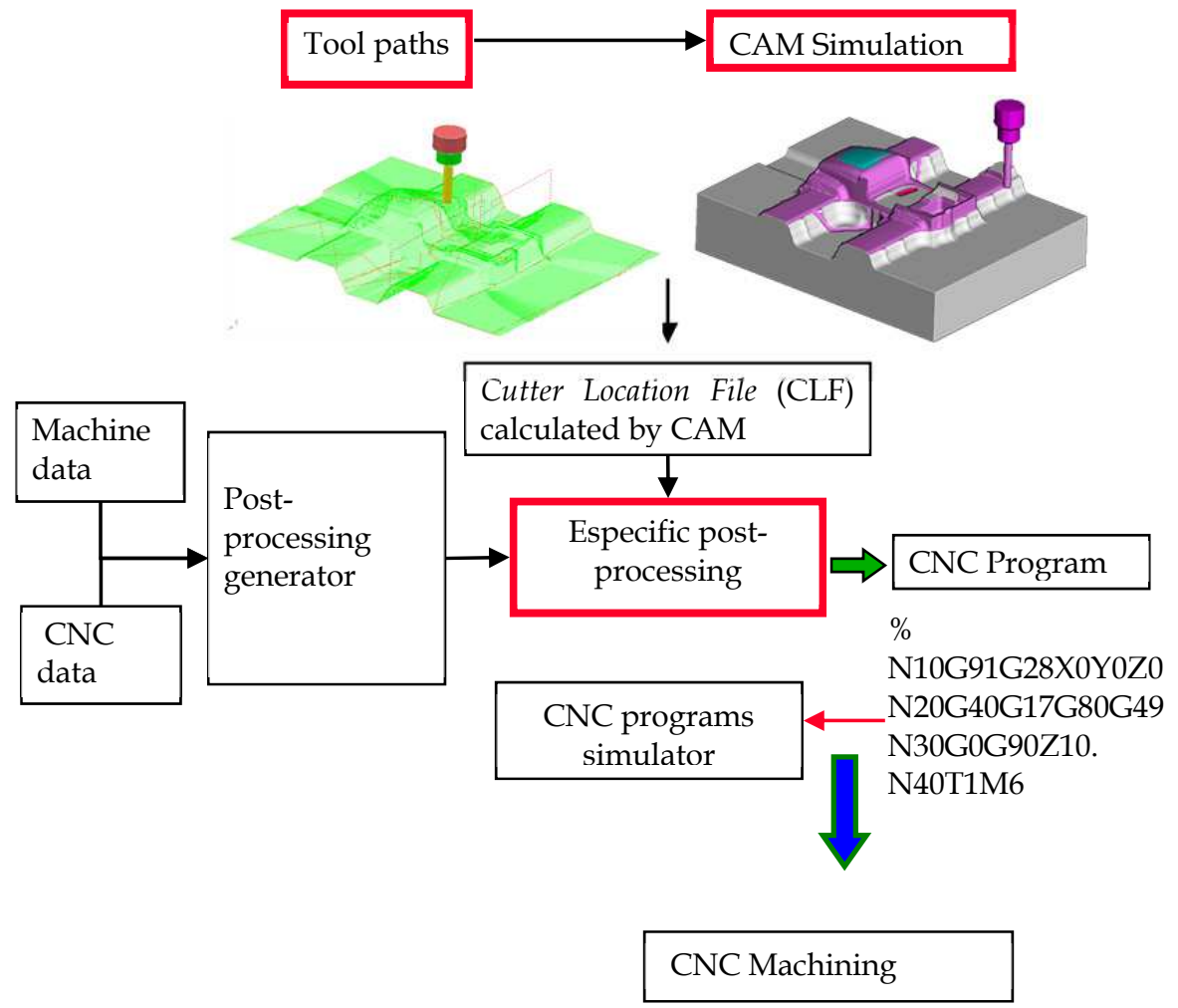

Fig. 18. Procedures required at this stage of post-processing generating CNC programs

\subsection{Evolution CAM systems. A brief presentation}

CAM systems had a significant development in the late 70's. In the 80's began the industrial deployment, allowing the programming of CNC machines via software, using CAD-CAM integration. However, with great limitations.

In the early 90's the development of hardware and software allowed the CAM systems could be run on personal computers. As a result, these systems have become more accessible for industry, which uses this technology successfully. Yet this time, can be mentioned some limitations in programming, such as:

- Errors in the calculated trajectories were common. Unnoticed damage could work (invading the piece), damage the tool or even the machine. Today, the calculated trajectories are much safer.

- Calculations of the trajectories were extremely slow. By the end of the 90s, even using sophisticated computers, for the time, calculations of trajectories with more than 8 hours in duration were not hard to find. It was the practice of some users to leave 
preparations for the calculation, several programs with the variable set and other items, and end of the day, requesting the initiation of the calculations (Bach processing). The computer performed the calculations at night to use the CNC program the next day. Processing times are reduced, allowing even the possibility of studying different paths to perform a specific machining.

- The machining simulation stage was also costly. On some occasions the simulation does not detect collisions. Today, in addition to more accurate simulations are faster (gouge free software).

- Great difficulty in limiting the work area when you wanted to perform machining in specific regions of a part, such as finishing or the use of a smaller diameter tool, for example. It was often necessary to use CAD to these limits (sometimes it is still convenient). These limits are known as borders or boundaries. Due to the difficulty in limiting areas of machining, the tool on many occasions it moved for a long time without removing material, to reach the region containing material to be removed. Current systems allow the creation of boundaries very efficiently.

- Software only for trajectories calculated for conventional geometries of the cutting tools. Currently, a range of tool geometries are available for calculating the trajectories.

- The variety of tool paths was extremely limited. There were few options for roughing and finishing. Today, the systems offer a wide variety of machining options, as the geometric shape. However, they still are not able to automatically identify the best machining strategies. This work is based on the user experience.

- The creation of a post-processor for a particular machine was not so simple. Today, although there are still challenges and specific problems, this step is already consolidated.

Even with these difficulties, the use of CAM systems provided a significant evolution in the CNC programming process for the manufacture of moulds, dies and parts containing complex geometric shapes. After the mid-90s, industries requiring complex machining and did not have a platform CAD / CAM / CNC, were doomed to failure.

Today, CAM systems, 3-axis milling, are evolving at a level reaching its apex, the trajectories of the calculations and simulation. A market trend is the application of milling for 5 degrees of freedom. In this operation, apart from the machine and $\mathrm{CNC}$, CAM system must also be able to calculate trajectories and simulation.

\section{Application of CAD systems}

A CAD (Computer Aided Design/Drafting) has been developing to assist the design and part modelling through interaction with the computer which defines all geometric information necessary for manufacture. Currently there are a large number of systems available in the market, ranging in a first classification between 2D and 3D systems.

It should be aware that a CAD system may be more suitable for specific applications, and many of these systems should not be considered competitors. This is because there are CAD systems to meet product development activities and geometrical design which has powerful tools for modelling complex shapes, as well as CAD systems that supply more design functions properly. It is common to find companies working with $3 \mathrm{D}$ CAD systems inadequate to the tasks assigned. 
To assist in the correct definition of a specific CAD platform for a given application, CAD systems can be classified as: i) Low-end 2D CAD systems, ii) Middle-end 3D CAD systems modelling of solid objects or surfaces, iii) High-end CAD hybrid system allows the modelling of solid objects and surfaces, among other features. A CAD solid or surface model refers to its core used for the mathematical representation of three-dimensional objects as shown below.

\subsection{CAD surface modelling - Middle end}

The surface model uses mathematical tools that allow creation of complex shapes with greater interactivity. The geometry created in a model of surfaces have no thickness, geometry is a "shell" and therefore no mass and centre of gravity (although it can be estimated). These are the main features of a CAD surface model. In CAD, the modelled objects may be closed. Surfaces are created using interpolation between the lateral and longitudinal representing the outline of the geometry. Although current CAD systems can identify regions of the opposite surface, this does not necessarily represent regions of an internal or external object.

The surface modelling CAD systems are able to perform the modelling of any geometric shape, regardless of their complexity. They have sophisticated features for manipulation of the geometry modelled by allowing the geometry to be changed interactively to obtain the desired product. The manipulation of the surface is given by the movement of any point of the geometry in any direction.

Besides this possibility, four are associated tangent vectors at each point on the surface. Vectors can be edited as the direction and magnitude. These devices allow changing any part of the surface. The possibility and ease of editing of any point of geometry, ensuring continuity of tangency and curvature, is an important feature that distinguishes the CAD surface model.

Figure 19 illustrates examples of issues in the geometric model. In this case, using point geometry (Figure a) there were three editions: Figure b) point geometry has moved in the vertical direction, $\mathrm{Y}$ axis in this case. It is observed that the geometry accompanying the extent varied. Figure c) pushed up the value of the magnitude of a vector. Figure d) has changed the angle of one of the vectors. Importantly, the changes in vector magnitude and slope can occur before and/or after the selected point.

It should be noted also that the edited point corresponds to an intersection between a lateral and longitudinal. So when it wants to change a point on the surface where there is no such intersection, the user must create lateral and longitudinal in order to obtain an intersection point. The creation of these entities is trivial in geometric surface models. However, the interactivity to identify their position is characteristic of each specific software, facilitating or hindering the work for the user.

The continuity of a surface can be considered in a simplified form as its degree of smoothness. Surfaces are called Class A high-quality surfaces in terms of continuity of tangency and curvature. This term is often used in the automotive industry (Souza \& Ulbrich, 2009). 


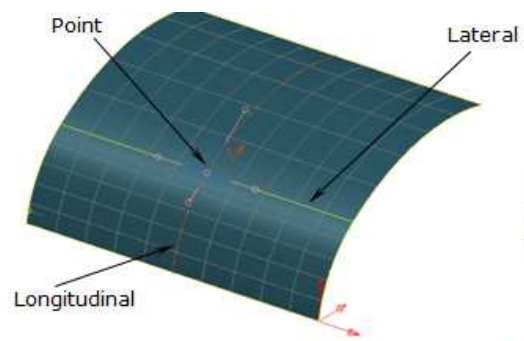

(a)

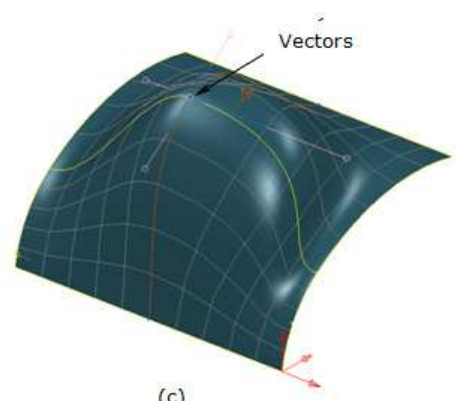

(c)

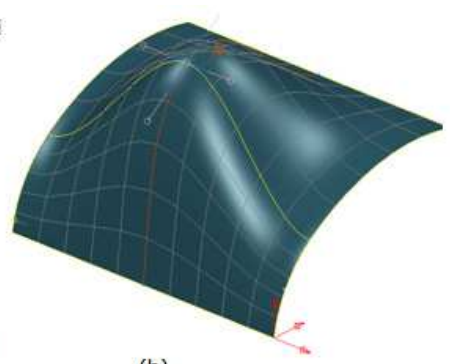

(b)

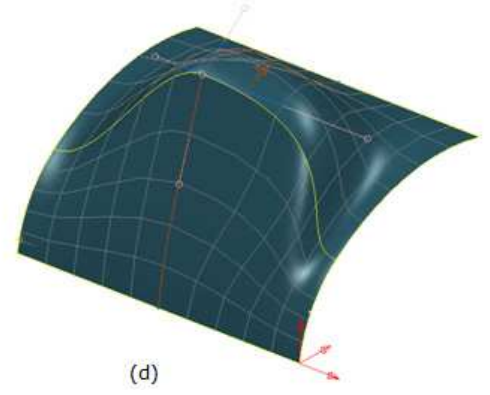

Fig. 19. Geometric edition on the surface

\subsection{Mathematics for constructing curves}

The quality of a curve is related to the mathematics used for this representation. Advanced CAD systems can offer different possibilities to generate a curve, in order to allow modelling of complex geometric forms, smooth and seamless.

Using parametric polynomial equations, Bezier-based methodology, the developers responsible for the development of each CAD software creates specific mathematical resources in order to allow greater control and ease of work to the user. This development of each software house is confidential and may represent the differential of the CAD software. Thus, only generic formulations of curves are discussed in the user guides for each software. Therefore, we will discuss below, some methodologies that differ from the creation of curves in different CAD systems.

Other mathematical formulations based on Bezier method have been developed for the representation of curves and surfaces in computer systems. The B-spline curve is also used for a parametric polynomial equation with modifications of the proposal Bezier, allowing among other things, to represent a curve using a polynomial of low degree, facilitating computations, also allowing local changes of the curve.

The Spline NURBS (Non-Uniform Rational B-Spline) represents the state of the art for the representation of curves and surfaces in CAD system, providing better control of the curve, allowing local issues, and allows more efficient calculation. Basically, the methodology is based on NURBS B-Spline method, adding two main functions: 
Non-Uniform: The vectors (knot) that indicate which portion of the curve is affected by a single point of control are not necessarily uniform.

Rational: You can set the intensity (weight) that each control point "attracts" the curve (exemplified below). It also allows the representation of elementary geometric entities: cylinders, cones, and plans, as well as conic curves, such as circles, ellipses, parabolas and hyperbolas.

In summary, these features mean more control factors can be applied to the curve, so more complex surfaces can be represented with a smaller number of turns, maintaining the continuity of curvature and tangency. For these reasons, the NURBS method is the state of the art in the representation of complex curves and surfaces in CAD systems.

The most advanced CAD systems can offer users different methodologies to create a curve. These methods are implemented by each developer and may involve from the choice between different mathematical functions, such as Bezier curves, B-Spline curve, NURBS curve, etc. As well as to allow changing the degree of the equation used.

It should be noted that each software has individual characteristics. The options of mathematical CAD software are not the same as other CAD software. These options, as well as construction tools and quality analysis of the geometries built, may be important differences for specific applications. These differences may involve the continuity of a curve, chances of building and editing, as well as computational workload.

The construction of curves in CAD systems is divided into two main mathematical methods:

a. Interpolation method. interpolation is to create a curve that passes through the points provided by the user. The curve of Figure 20 shows the interpolation process to create a curve, using four points as an example: P1 (0, 0), P2 (50, 50), P3 (100, -50), P4 (150, 0). The Hermite curve is a curve described by the example of interpolation method.

b. Approach method. In this case, the points provided by the user are used to create the control polygon will represent the curve, as shown in Figure 20 curve b. The Bezier curve is a curve described by the example of approximation method.

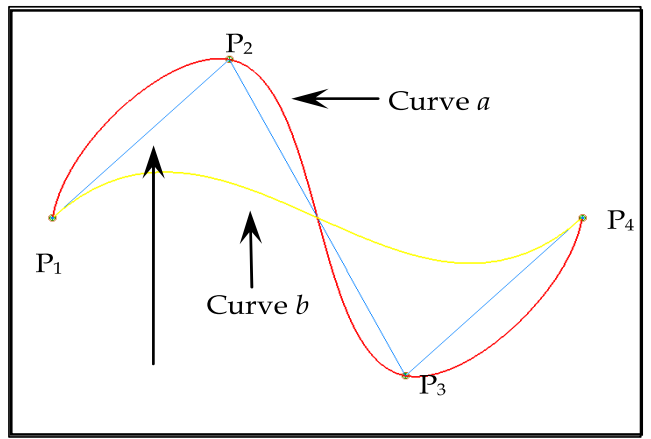

Fig. 20. Curves created by the methods of interpolation and approximation of points

Besides the method, interpolation or approximation, CAD systems can still provide other resources for modelling curves, such as: 
a. Degree of mathematical equation. Some CAD systems allow changing the degree of the equation used to construct the curve. Using the points P1 $(0,0)$, P2 $(50,50)$, P3 (100, $50), P 4(150,0)$, curves were generated by changing the degree of the polynomial, as shown in Figure 21. Note that due to the number of control points, according to the mathematical rule are allowed to generate curves of maximum degree 3 (the degree of the equation is 1 minus the number of control points). Also note that a polynomial of degree 1 generates a sequence of lines.

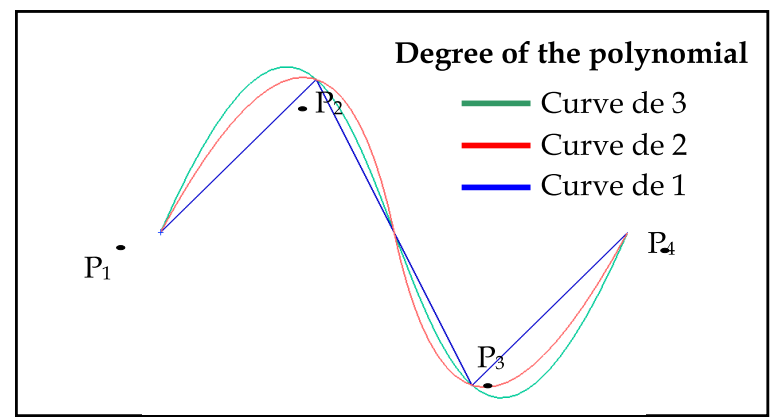

Fig. 21. Curves constructed by polynomials of different degrees

b. Different equations. Some CAD systems do not allow changing the degree of the polynomial used to construct the curve. However, many systems offer different equations to build a curve, as illustrated in Figure 22.

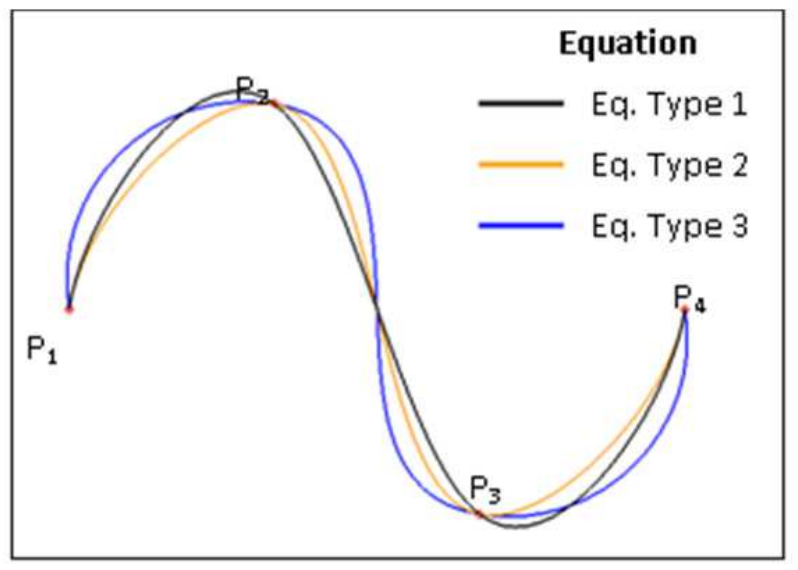

Fig. 22. Curves for different mathematical built using the same points

It is important to note that the same sequence of points can build distinguish ways in the same CAD system. Thus, it is difficult to build the same geometrical shape in two different CAD systems using the same sequence of points.

c. Influence of the control points (weight). Some CAD systems allow changing the degree of influence of each control point of the polygon under the curve calculated (Figure 23). 


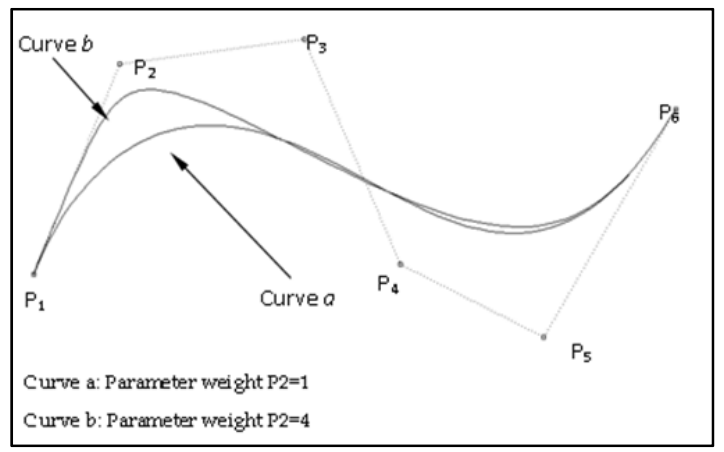

Fig. 23. Changed the influence of the point P2 on the curve

These are resources that help advanced modelling of complex geometries containing forms.

\subsection{Curvature continuity}

The continuity of geometry is one way to assess the quality of a geometric entity generated by CAD software. The level of continuity of geometry is related to the degree of smoothness along its length. Geometry can represent discontinuous aesthetic and functional implications in a product. The continuity of surfaces is a concept used to extrapolate the curves. Thus, it is used the concepts of continuity of curves to present and discuss this topic. A discontinuous curve may generate a discontinuous surface. The continuity of a geometry, curve or surface, can happen in two cases:

a. Going over a single entity.

b. Continuity in the connection between two distinct entities.

There are three size classes for the continuity of curves and surfaces.

1. Continuity of position: occurs when two segments of the same entity or two separate entities, have in common only the point of contact (Figure 24b). This is the case of less smoothness.

2. Continuity of position and tangent: occurs when a point between the segments analyzed, or two separate entities, have in common other than the contact tangent vectors (Figure 24c).

3. Continuity of position, tangency and curvature: occurs when a point between the segments analyzed, or two different geometries have in common, the contact point, tangent vector and the same degree of curvature (Figure 24d). This is the case for better continuity.

Figure 24 illustrates the case for continuity in the connection between two different curves, connected by a user or other mechanism of software construction, as in the construction of borders to make cuts at the intersection of surfaces. These boundaries are spline curves calculated by the software and have degree of continuity. The quality of this curve can influence the surface generated by it.

As mentioned, the continuity can occur along a single curve, depending on the model used for its representation. 
This can partition the curve internally into segments known as patches, and it often is not noticed by the user.

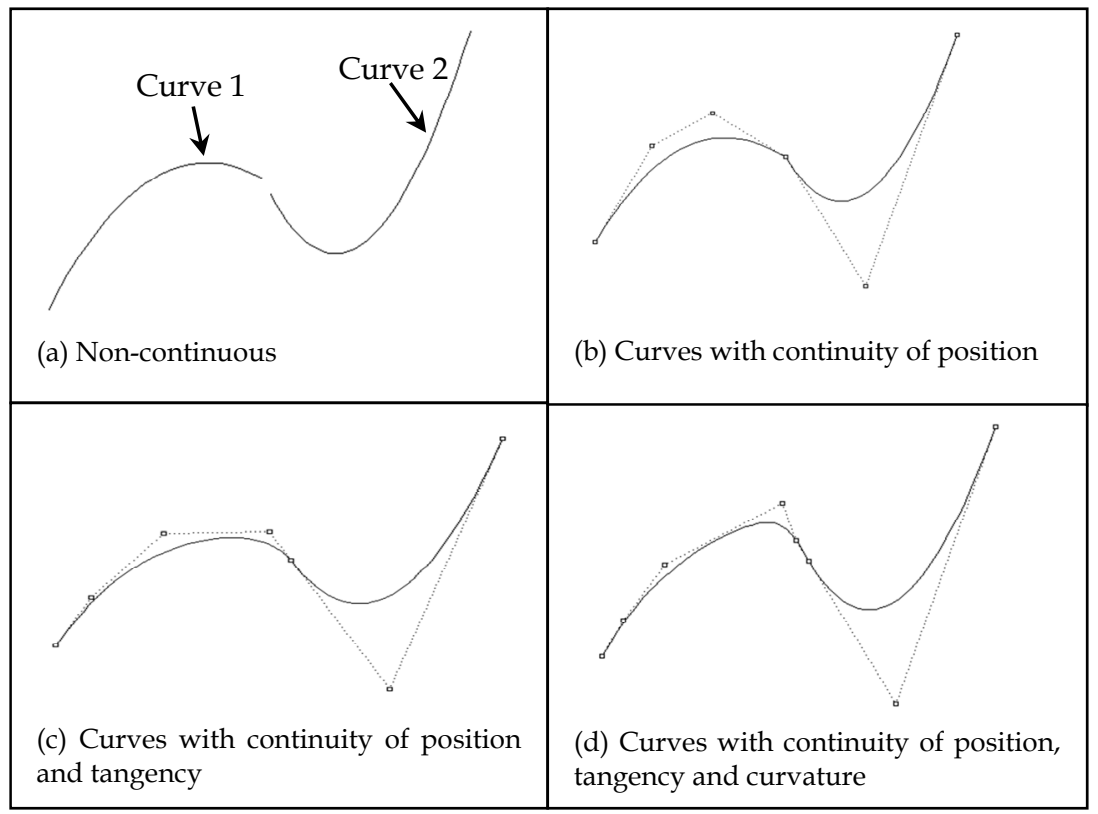

Fig. 24. Degree of continuity of continuity of a curve

\subsection{Quality of surfaces generated by CAD systems}

In many cases it is necessary for modelling three-dimensional boundary conditions that go beyond the requirements of design and ergonomics. Surfaces are required in these cases with a high degree of continuity in order to maintain the smoothness in the shape of the product. The quality of a complex surface generated by a CAD system is related to the model used for its creation in three circumstances:

a. The smoothness of a surface can be related to the continuity of the curve used for its creation.

b. The connection between different areas may represent regions with impaired smooth.

c. Areas of intersection with rays (Fillets) calculated "automatically" by the software.

When you want to develop surfaces for products requiring strict-looking, such as appliances, the external body of a car, etc., small discontinuities in the surfaces of the 3D CAD model can be replicated in the final product, be it cast or stamped. When creating surfaces through the interpolation of two sections and non-planar curves with different radius of curvature, and the need to maintain continuity of the tangential surface created in which the directions of the surface change smoothly, the surface must also have continuity of curvature. Applying tests of light reflection on the product such geometric discontinuities can be found. 
CAD systems have advanced graphical tools to detect such discontinuities in a surface. Figure 25b illustrates a surface with discontinuities. The CAD system identifies the discrete regions through hazards. The parallel strips and gently represent regions consistent with good degree of continuity. The discrete regions are illustrated by the tracks with abrupt changes (Figure 25c). This may occur for mathematical limitations and / or the method used to construct the surface. The surface of Figure 25 (b and c) is a surface Fillet, calculated automatically by the software.
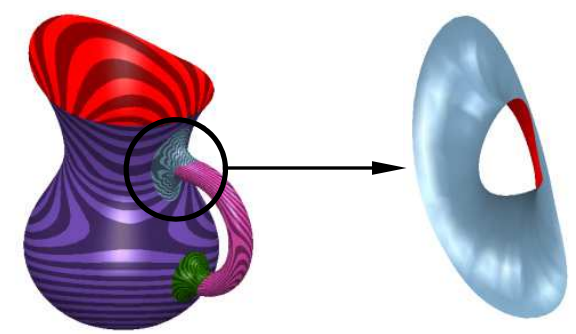

(b) Discontinuous surface

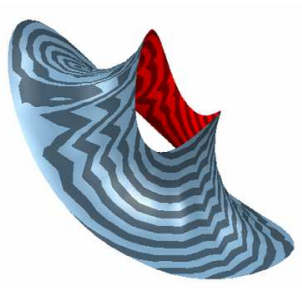

(c) Abrupt changes in gray bands

Fig. 25. Surface emphasized the problem of discontinuity

The abrupt changes of the bands indicate regions with continuity compromised. However, after identifying these regions and/or surfaces, CAD systems are still not able to help troubleshoot the problem. The reasons that caused specific discontinuities are not presented.

Thus, the user must develop empirical ability to solve or minimize the problem. In the case illustrated in Figure 25, the surface of agreement (Fillet) was calculated by the software with a large number of longitudinal and lateral, creating the problem of continuity observed. Performing a work setting "manual" to delete longitudinal and lateral, it was observed an improvement in continuity of surface.

\subsection{CAD solid modelling - Middle end}

The mathematics used in the solid model ensures the reality of constructive geometric entity modelled. The solid model performs mathematical methods to validate real possibility of the existence of the modelled geometry. Because of this geometric validation, among other factors, a CAD solid model is great limitations to the modelling of complex shapes, such as, for example, a computer mouse, the panel of a car, bottle packaging, among others objects. There are difficulties in parts of issues surface in the tangent of specific points and ensuring continuity. To work with geometries with these characteristics, a CAD modeller surfaces may be more appropriate.

A CAD solid model should possess the following characteristics:

- Geometry unambiguous and real.

- Generation of simple objects (compared to the models of surfaces).

- Suitable for engineering geometry. 
- Cost less than the CAD surface model.

- Limited to create complex geometric shapes.

- Use relatively simple.

Different computational techniques for solid modelling. The internal representation of a solid object is commonly used to classify the modeller. The internal representation is how the software stores the model. This is different from the external representation, which presents the model on the computer screen. Two techniques of internal representation are: a) B-Rep (Boundary Representation). This representation employs models described by boundaries, proposed by Ian Braid, Cambridge University. b) CSG (Constructive Solid Geometry). This method was proposed by Voelcker and Requicha the University of Rochester.

Both methods were applied in commercial systems in the late 70's. Other methods of representation of solids were developed, such as octree, quadtree, cell breakdown, among others. However, the methods of representation of solids used in most current CAD systems are the CSG (Constructive Solid Geometry) and B-Rep (Boundary Representation).

In this method, solid objects are built with a combination of simple solid primitives such as cylinders, cubes, cones, spheres, etc. Through Boolean operations (George Boole, 1815-1864, developed the Boolean algebra) addition, subtraction and intersection between solid objects in case of CAD software. When an object is constructed with a CSG modeller, the system saves a history of creation (creating topographic tree), which includes all solid primitives with their size and positioning, and information about all the Boolean operations used to construct the object.

\subsection{CAD hybrid modelling - High end}

The hybrid model allows building three-dimensional geometries employed the techniques of combining modelling surfaces in solid modelling. This provides greater versatility to work for the user, can benefit from the best features of both methods.

\subsection{Techniques of modelling}

There are three main techniques, common in CAD systems for the construction of threedimensional geometries: i) primitive geometries ii) intersecting geometries iii) domain-based wireframe geometries. The latter will be the most used, in this case the objects are constructed using geometric information in wireframe, and may be straight lines, curves, arcs, points or a combination thereof. The software uses this field and applies the function selected by the user, which are: operation of revolution or extrusion of a profile on a directional vector; extrusion of a profile on another profile directional; interpolation between curves; migration between cross sections. More complex surfaces, which require patches with three or five boundaries, are more complex representation. These surfaces are found in regions with corners at the intersection of surfaces, as well as the junction of three Fillets.

\section{Application of CAE systems}

The Computer Aided Engineering (CAE) systems were developed to simulate real applications, and enabling auxiliary steps of projects and manufacturing. There exists CAE 
software specifically for each application. The software SigmaSoft ${ }^{\circledR}$ and Moldflow ${ }^{\circledR}$ are examples of CAE to simulate the transformation of plastic and none metallic products. The software Ansys ${ }^{\circledR}$ and Nastran ${ }^{\circledR}$ are more specific for simulations of mechanical demands dynamic statistics.

Simulations of products and processes have been highly desired for the last decade for various applications. Secure results of these simulations can prevent accidents, reduce manufacturing costs, optimise products behind for example the reduction of the thickness, how to help in the substitution of materials, collecting materials, and making processes and products more efficient.

The CAE systems make sophisticated mathematical algorithms to perform calculations. The quality of a simulation involves a degree of approximation between the results obtained and the real situation. This relation of the mathematical algorithm employed for the generation of the finite elements mesh and for the calculations asked for. This way the information entered, such as the geometry, mechanical demands and materials.

Information about the process to be simulated must be attributed to the CAE system to make the calculations. This information is called entrance variables. Both variables are specifically defined in function of the process and/or the product studied.

To carry out the simulation calculations, CAE uses finite technical elements. The geometry originating from CAD can be used to analyse the primitive elements, such as triangles. Therefore the software generates a geometry dominated by a finite elements mesh, which represents an object or a process to be simulated. Finite elements and methods are widely employed by CAE systems.

The working procedures to carry out simulations with CAE systems can be divided in three main phases: pre-processing (generation of the sweater of finite elements and variables of entrance), processing (calculation of the demands) and post-processing (evaluation and interpretation of the answer of the software), approached with more property in the sequence.

In the pre-processing the modelling of the product is supplied by the CAD system. In case it cannot be integrated with the systems CAD/CAE, the geometry should be exported by the CAD through a standard interface (IGES, by example) and imported in the CAE system. After importing the geometry, the CAE system should calculate the finite elements mesh. This is a crucial step for the quality of simulation, because the nodes of the finite elements mesh are used as the basis for all calculations performed in CAE applications.

The quality of the finite elements mesh generated by the software is attributed mainly by the homogeneity of the elements that it composes. The best condition will be generated by software that contains the largest number of elements and dimensions equilateral homogeneous. The mesh calculated by the software can at times be unstable, having repercussions in future simulations. In these cases the user should be qualified to identify and correct such limitations of software, which can be related with the complexity of the surface, rank of refinement of the mesh, quality of the surface imported of the CAD via communication patterns, beyond the mathematical limitations made for generation of the mesh. Figure 26 (a) illustrates the CAD model, (b) example of mesh with none equilateral regions containing elements and a fault of connection between the elements, which represents errors or impossibilities of calculation. 


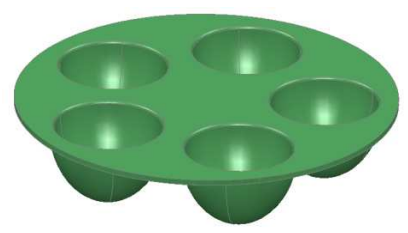

(a)

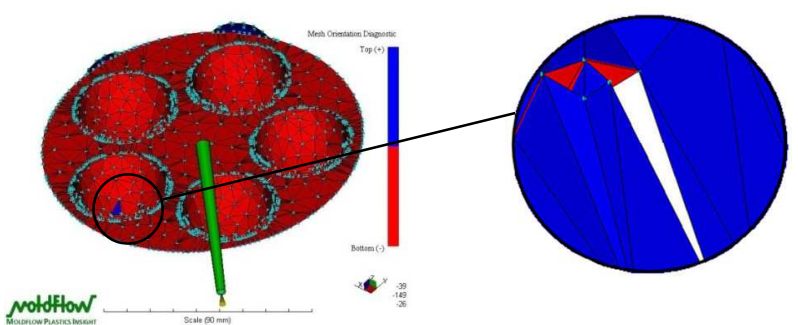

(b)

Fig. 26. Finite elements mesh with problems. a) Product CAD; b) Mesh. Plans not oriented and non-equilateral triangles; open mesh

In cases of problems in the automatic construction of the finite elements mesh, the user should interact with the software in order to provide an adequate mesh. The dimensions of the elements that compose the mesh can be defined by the user and will have repercussions in the precision of the calculation. Still in the pre-processing, all the conditions of contour should be supplied, such as: mechanical stresses, strength of the product material, temperature, among other information that composed the simulation.

The results, in general, are presented in the form of multicoloured graphics, according to the analysis requested. Figure 27 illustrates the result of the simulation of the injection of a plastic product, having like variables in the temperature injection necessary, the time of injection of the product, and the pressure of injection.

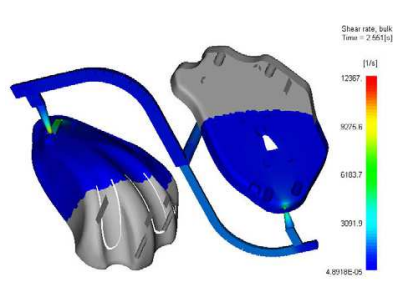

(a) Temperature

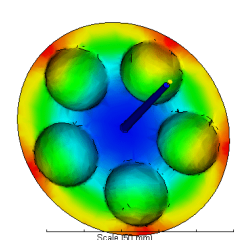

(b) Cycle time

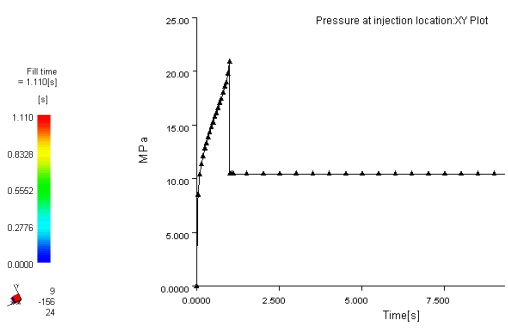

(c) Pressure of injection

Fig. 27. Simulation results of the injection process

In this case it is also possible to simulate the cooling systems of the mould and the degree of efficiency of each proposal.

\section{Application of CAI systems}

The Computer-Aided Inspection (CAI) represents the final integration of manufacturing processes, this step is performed as a comparison between the geometric model constructed in the CAD, which represents the theoretical form of the design geometry without dimensional errors, with the product manufactured, which has so errors arising from mechanical limitations of both machine tool and manufacturing process. 
At this stage, the CAI software receives the geometric information of CAD and information representing the final product, obtained by a coordinate measuring machine, through a cloud of points. CAI compares the geometric errors between the real object with the CAD design and report on the regions with the geometrical deviations, considering the tolerances specified in the project (Figure 28).
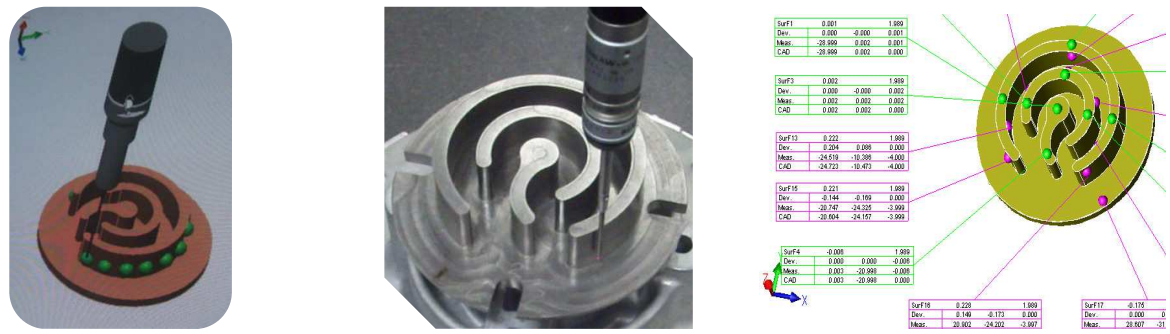

Fig. 28. Integration of CAD/CAI/MMC

\section{Acknowledgments}

The authors would like to thank CAPES for supporting this research project under the grant PROCAD 139/2007 and PROENGENHARIAS PE 27/2008. The authors also would like to thank the research group PROMOLDE from IST/SOCIESC, Villares Metals for supplying the raw material and Sandvik Corromant.

\section{References}

Coelho, R. T.; Souza, A. F.; Rodrigues, A. R. (2009). Mechanistic approach to predict real machining time for milling free form geometries applying high feed speed. International Journal of Advanced Manufacturing Technology, v. 1, p. 1433-30151433-3015.

Souza, A. F.; Coelho, R. T, (2007). “Experimental Investigation of Feed Rate Limitations on High Speed Milling Aimed at Industrial Applications". Int. Jou. Adv. Manufacturing Technology. DOI 10.1007/s00170-006-0445-2.

Souza, A. F.; Coelho, R. T.; Rodrigues, A. R. (2010). Manufacturing complex geometries using high speed cutting technology. VDM Verlag. v. 1. 130 p.

Souza, A. F.; Coelho, R. T. (2007). Investigation of Spline polynomial for describing a free form tool path for high speed milling. In: International Conference on High Speed Machining. San Sebastian, Spain.

Souza, A. F.; Ulbrich, C. B. L. (2009). Engenharia Integrada por Computador e Sistemas CAD/CAM/CNC. Princípios e Aplicações. 1. ed. São Paulo: Artliber. v. 1. 332p.

Souza A.F. (2004). Contribution for free form milling applying the High Speed Cutting technology. Doctored thesis. University of Sao Paulo. 186pp.

Yau, H.T.; Kuo, M.J. (2001). NURBS machining and feed rate adjustment for high-speed cutting of complex sculptured surfaces. International Journal of Production Research, London, v.39, n.1, p.21-41, Jan.

Elbestawi, M.A. (1997). High-speed milling of dies and molds in their hardened state. Annals of the CIRP, Paris, v.46, n.1, p.57-62. 


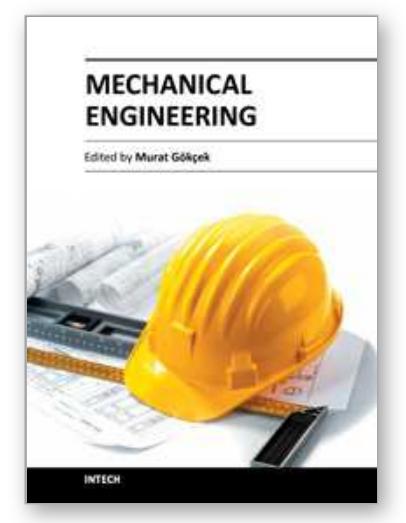

\author{
Mechanical Engineering \\ Edited by Dr. Murat Gokcek
}

ISBN 978-953-51-0505-3

Hard cover, 670 pages

Publisher InTech

Published online 11, April, 2012

Published in print edition April, 2012

The book substantially offers the latest progresses about the important topics of the "Mechanical Engineering" to readers. It includes twenty-eight excellent studies prepared using state-of-art methodologies by professional researchers from different countries. The sections in the book comprise of the following titles: power transmission system, manufacturing processes and system analysis, thermo-fluid systems, simulations and computer applications, and new approaches in mechanical engineering education and organization systems.

\title{
How to reference
}

In order to correctly reference this scholarly work, feel free to copy and paste the following:

Adriano Fagali De Souza and Sabrina Bodziak (2012). Advanced Free Form Manufacturing by Computer Aided Systems - Cax, Mechanical Engineering, Dr. Murat Gokcek (Ed.), ISBN: 978-953-51-0505-3, InTech, Available from: http://www.intechopen.com/books/mechanical-engineering/advanced-free-form-manufacturingby-computer-aided-systems-cax

\section{INTECH}

open science | open minds

\author{
InTech Europe \\ University Campus STeP Ri \\ Slavka Krautzeka 83/A \\ 51000 Rijeka, Croatia \\ Phone: +385 (51) 770447 \\ Fax: +385 (51) 686166 \\ www.intechopen.com
}

\author{
InTech China \\ Unit 405, Office Block, Hotel Equatorial Shanghai \\ No.65, Yan An Road (West), Shanghai, 200040, China \\ 中国上海市延安西路65号上海国际贵都大饭店办公楼 405 单元 \\ Phone: +86-21-62489820 \\ Fax: $+86-21-62489821$
}


(C) 2012 The Author(s). Licensee IntechOpen. This is an open access article distributed under the terms of the Creative Commons Attribution 3.0 License, which permits unrestricted use, distribution, and reproduction in any medium, provided the original work is properly cited. 